\title{
Besicovitch pseudodistances with respect to non-Følner sequences
}

\author{
Silvio Capobianco*† $\quad$ Pierre Guillon ${ }^{\ddagger} \quad$ Camille Noûs ${ }^{\S}$
}

\begin{abstract}
The Besicovitch pseudodistance defined in BFK99 for one-dimensional configurations is invariant by translations. We generalize the definition to arbitrary groups and study how properties of the pseudodistance, including invariance by translations, are determined by those of the sequence of finite sets used to define it. In particular, we recover that if the Besicovitch pseudodistance comes from a nondecreasing exhaustive Følner sequence, then every shift is an isometry. For non-Følner sequences, we prove that some shifts are not isometries, and the Besicovitch pseudodistance with respect to some subsequence even makes them non-continuous.
\end{abstract}

Keywords: Besicovitch distance, Følner sequences, submeasures, amenability, non-compact space, symbolic dynamics.

\section{Introduction}

The Besicovitch pseudodistance was proposed by Blanchard, Formenti and Kưrka in BFK99 as an "antidote" to sensitivity of the shift map in the prodiscrete (Cantor) topology of the space of $1 \mathrm{D}$ configurations over a finite alphabet. The idea is to take a window on the integer line, which gets larger and larger, and compute the probability that in a point under the window, chosen uniformly at random, two configurations will take different values. The upper limit of this sequence of probabilities behaves like a distance, except for taking value zero only on pairs of equal configurations: this defines an equivalence relation, and the resulting quotient space is a metric space on which the shift is an isometry, or equivalently, the distance is shift-invariant.

The original choice of windows is $X_{n}=[-n: n]$, the set of integers from $-n$ to $n$ included. This notion can be easily extended to arbitrary dimension $d \geq 1$, taking a sequence of hypercubic windows. If we allow arbitrary shapes, the notion of Besicovitch space can be extended to configurations over arbitrary groups; in this case, however, the properties of the group and the choice of the windows can affect the the distance being or not being shift-invariant. An example of a Besicovitch pseudodistance which is not shift-invariant is given in [Cap09], where it is also proved that, if a countable group is amenable (cf. [CGK13] and [CSC10, Chapter 4]), then the Besicovitch distance with respect to any nondecreasing exhaustive Følner sequence is shift-invariant. The class of amenable groups is of great interest and importance in group theory, symbolic dynamics, and cellular automata theory.

In this paper, we explore the relation between the properties of Besicovitch pseudodistances over configuration spaces with countable base group and those of the sequence of finite sets

\footnotetext{
*Department of Software Science, Tallinn University of Technology. Silvio@cs.ioc.ee, silvio.capobianco@taltech.ee

TThis research was supported by the Estonian Ministry of Education and Research institutional research grant no. IUT33-13.

${ }^{\ddagger}$ CNRS, Aix-Marseille Universit, Institut de Mathématiques de Marseille. pguillon@math.cnrs.fr

${ }^{\S}$ Laboratoire Cogitamus. camille.nous@noussommesluniversite.fr
} 
used to define it. In Section 3, we give the main definition and prove that if a sequence of finite subsets is increasing, then the corresponding Besicovitch space is pathwise connected: this generalizes [BFK99, Proposition 1]. In Section 4, we introduce a notion of synchronous Følner equivalence between sequences, and a related order relation where one sequence comes before another sequence if it is synchronously Følner-equivalent to a subsequence of the latter. This, on the one hand, generalizes that of Følner sequences, and on the other hand, allows us to compare the Besicovitch distances and submeasures associated to different sequences. In particular, we prove that an increasing sequence of finite sets is Følner if and only if every shift is an isometry for the corresponding Besicovitch distance: this provides the converse of Cap09, Theorem 3.5]. Finally, we give conditions for absolute continuity and Lipschitz continuity of Besicovitch submeasures with respect to each other.

\section{Background}

We use the notation $X \Subset Y$ to mean that $X$ is a finite subset of $Y$. We denote the symmetric difference of two sets $X$ and $Y$ as $X \Delta Y$.

Given $\alpha \in \mathbb{R}$, its integer part $\lfloor\alpha\rfloor$ is the largest $m \in \mathbb{Z}$ such that $m \leq \alpha$.

If $\left(\alpha_{n}\right)$ and $\left(\beta_{n}\right)$ are nonzero number sequences, we write $\alpha_{n} \sim_{n \rightarrow \infty} \beta_{n}$ if $\lim _{n \rightarrow \infty} \alpha_{n} / \beta_{n}=1$, and $\alpha_{n}=o_{n \rightarrow \infty} \beta_{n}$ if $\lim _{n \rightarrow \infty} \alpha_{n} / \beta_{n}=0$.

\section{$2.1 \quad$ Submeasures}

The following definition appears for instance in [Sab06].

Definition 2.1. A submeasure over a set $G$ is a map $\mu: 2^{G} \rightarrow \mathbb{R} \sqcup\{+\infty\}$ such that:

1. $\mu(\emptyset)=0$;

2. $\mu(W)<\infty$ if $W$ is finite;

3. $\mu(V \cup W) \leq \mu(V)+\mu(W)$ for every $V, W \subset G$.

If $G$ and $A$ are two sets, the difference set of two functions $x, y: G \rightarrow A$ is the set $\Delta(x, y)=$ $\{i \in G \mid x(i) \neq y(i)\}$.

Any submeasure over $G$ gives rise to an associated pseudodistance over $A^{G}$ :

$$
d_{\mu}(x, y)=\mu(\Delta(x, y)) \forall x, y \in A^{G} .
$$

Remark 2.2. The topological space corresponding to such a pseudodistance is homogeneous in the following sense: the balls around every two points $y$ and $z$ are isometric. Indeed, identify $A$ with the additive group $\mathbb{Z} /|A| \mathbb{Z}$. Then for every $y, z \in A^{G}$ the map $\psi_{y, z}: A^{G} \rightarrow A^{G}$ defined by $\psi_{y, z}(x)(i)=x(i)-y(i)+z(i)$ for every $x \in A^{G}$ and $i \in G$ is an isometry between any ball around $y$ and the corresponding one around $z$.

We say that submeasure $\mu$ is absolutely continuous (resp. $\alpha$-Lipschitz, for some $\alpha>0$ ) with respect to submeasure $\nu$ if $\nu(W)=0 \Longrightarrow \mu(W)=0$ (resp. $\mu(W) \leq \alpha \nu(W)$ ) for any $W \subset G$.

Remark 2.3. Let $\varepsilon, \delta>0, \mu, \nu$ two submeasures on $G$, and $z \in A^{G}$. The following are equivalent.

1. For every set $W \subset G, \mu(W) \geq \varepsilon \Longrightarrow \nu(W) \geq \delta$.

2. For every $x, y \in A^{G}, d_{\mu}(x, y) \geq \varepsilon \Longrightarrow d_{\nu}(x, y) \geq \delta$. 
3. For every $x \in A^{G}, d_{\mu}(x, z) \geq \varepsilon \Longrightarrow d_{\nu}(x, z) \geq \delta$.

Consequently, the identity map, from space $A^{G}$ endowed with $d_{\mu}$ onto space $A^{G}$ endowed with $d_{\nu}$, is continuous (resp. $\alpha$-Lispchitz) if and only if $\mu$ is absolutely continuous (resp. $\alpha$-Lispchitz) with respect to $\nu$. In that case the identity is even absolutely continuous.

\subsection{Shifts and translations}

If $A$ is an alphabet, $G$ is a group, and $g \in G$, the shift by $g$ is the function $\sigma^{g}: A^{G} \rightarrow A^{G}$ defined by $\sigma^{g}(x)(i)=x(g i)$ for every $x \in A^{G}$ and $i \in G$. A map $\psi$ from $A^{G}$ to itself is shift-invariant if $\psi \sigma^{g}=\sigma^{g} \psi$ for every $g \in G$. Note that $\Delta\left(\sigma^{g}(x), \sigma^{g}(y)\right)=g^{-1} \Delta(x, y)$ for every $x, y \in A^{G}$ and $g \in G$, as:

$$
\begin{aligned}
\Delta\left(\sigma^{g}(x), \sigma^{g}(y)\right) & =\{i \in G \mid x(g i) \neq y(g i)\} \\
& =\left\{g^{-1} j \mid j \in G, x(j) \neq y(j)\right\} \\
& =g^{-1}\{j \in G \mid x(j) \neq y(j)\} \\
& =g^{-1} \Delta(x, y) .
\end{aligned}
$$

Since the maps $\psi_{y, z}$ from Remark 2.2 are shift-invariant, one can see that the shift is continuous, Lipschitz, etc in every $x$ if and only if it is in one $x$.

The shift by $g$, within space $A^{G}$ endowed with $d_{\mu}$, is topologically the same as the identity map, from $A^{G}$ endowed with $d_{\mu}$ onto space $A^{G}$ endowed with $d_{\nu}$, where $\nu(W)=g^{-1} \mu(W)=$ $\mu\left(g^{-1} W\right)$ for any set $W \subset G$. Remark 2.2 can then be rephrased into the following.

Remark 2.4. If $G$ is a group, $g \in G$, and $A^{G}$ is endowed with $d_{\mu}$, then the shift map by $g$ is continuous (resp. $\alpha$-Lispchitz) if and only if $\mu$ is absolutely continuous (resp. $\alpha$-Lispchitz) with respect to $g^{-1} \mu$. In that case, the shift by $g$ is even absolutely continuous.

\section{Besicovitch submeasure and pseudodistance}

Among classical examples of submeasures are the ones that induce the Cantor topology, or shift-invariant Besicovitch, or Weyl pseudodistance (see [HM17, Def 4.1.1]. We will focus on the Besicovitch topology.

\subsection{Definition}

Let $X$ and $Y$ be nonempty sets and let $\left(X_{n}\right)$ be a nondecreasing sequence of finite subsets of $X$. We may or may not require that $\left(X_{n}\right)$ be exhaustive, that is, $\bigcup_{n} X_{n}=X$.

Let us denote $\mathfrak{P}(W \mid V)=\frac{|W \cap V|}{|V|}$ (by convention, this is $+\infty$ if $V=\emptyset$ ).

\section{Remark 3.1.}

1. $\mathfrak{P}(W \cup U \mid V) \leq \mathfrak{P}(W \mid V)+\mathfrak{P}(U \mid V)$, and the equality holds if the union is disjoint.

2. If $V \subset U$, then $\mathfrak{P}(V \mid U) \mathfrak{P}(W \mid V)=\mathfrak{P}(V \cap W \mid U) \leq \mathfrak{P}(W \mid U)$

The Besicovitch submeasure $\mu_{\left(X_{n}\right)}: 2^{X} \rightarrow[0,1]$ is defined by:

$$
\mu_{\left(X_{n}\right)}(W)=\limsup _{n} \mathfrak{P}\left(W \mid X_{n}\right) .
$$

The Besicovitch pseudodistance is $d_{\left(X_{n}\right)}=d_{\mu_{\left(X_{n}\right)}}$. For example, if $X=\mathbb{N}, Y=\{0,1\}$, and $X_{n}=[0: n-1], x(i)=0$ for every $i \in \mathbb{N}$ and $y \in\{0,1\}^{\mathbb{N}}$ is the characteristic function of the prime numbers, then $d_{\left(X_{n}\right)}(x, y)=0$.

The topology of the quotient space is very different from the prodiscrete (Cantor) topology. 
Remark 3.2. Remark obviously two dual cases (in general we will be in the first case, but not the second one):

1. If every $U \Subset G$ appears finitely many times in $\left(X_{n}\right)$, then $\mu_{\left(X_{n}\right)}(W)=0$ if $W$ is finite.

2. If all $U \Subset G$ appear (or, more generally, if for every $n$, cofinitely many $U \Subset G$ of cardinality $n$ appear) in $\left(X_{n}\right)$, then $\mu_{\left(X_{n}\right)}(W)=1$ if $W$ is infinite.

We will now concentrate on the nondecreasing case.

\subsection{Connectedness}

Theorem 3.3. If $\left(X_{n}\right)$ is nondecreasing and has unbounded cardinality, then the Besicovitch space is pathwise-connected.

Proof. It is enough to build, for every $W \subset G$ and for every $\alpha \in[0,1]$, a set $V(\alpha)$ such that:

1. if $0 \leq \alpha<\beta \leq 1$ then $V(\alpha) \subset V(\beta)$,

2. $V(0)=\emptyset$ and $V(1)=W \cap \bigcup_{n} X_{n}$ (the submeasure does not account for what is outside the union), and

3. $\mu(V(\alpha))=\alpha \mu(W)$.

Let us assume that there is a total order on each $Y_{n}=X_{n} \backslash \bigcup_{i<n} X_{i}$ (independent of our $\alpha$ ). Now define, inductively on $n \in \mathbb{N}$, the set $U_{n} \subset W \cap Y_{n}$ by taking the minimal $\left\lfloor\alpha\left|W \cap X_{n}\right|\right\rfloor-$ $\sum_{i<n}\left|U_{i}\right|$ elements in $W \cap Y_{n}$. Provided that this definition is valid, it maintains, for all $n \in \mathbb{N}$, the property that $\sum_{i \leq n}\left|U_{i}\right|=\left\lfloor\alpha\left|W \cap X_{n}\right|\right\rfloor$. It is actually valid because, by induction,

$$
\begin{aligned}
\left\lfloor\alpha\left|W \cap X_{n+1}\right|\right\rfloor-\sum_{i \leq n}\left|U_{i}\right| & =\left\lfloor\alpha\left|W \cap X_{n+1}\right|\right\rfloor-\left\lfloor\alpha\left|W \cap X_{n}\right|\right\rfloor \\
& <\alpha\left(\left|W \cap X_{n+1}\right|-\left|W \cap X_{n}\right|\right)+1 \\
& <\alpha\left(\left|W \cap Y_{n+1}\right|\right)+1 \\
& <\left|W \cap Y_{n+1}\right|+1 .
\end{aligned}
$$

We now define $V(\alpha)=\bigsqcup_{n \in \mathbb{N}} U_{n}$. By construction, thanks to the common total order, we immediately get that $\alpha<\beta \Longrightarrow V(\alpha) \subset V(\beta)$. Moreover,

$$
\begin{aligned}
\mu(V(\alpha)) & =\limsup _{n \in \mathbb{N}} \mathfrak{P}\left(V(\alpha) \mid X_{n}\right) \\
& =\limsup _{n \in \mathbb{N}} \frac{\left|\bigsqcup_{i \leq n} U_{i}\right|}{\left|X_{n}\right|} \\
& =\limsup _{n \in \mathbb{N}} \frac{\left\lfloor\alpha\left|W \cap X_{n}\right|\right\rfloor}{\left|X_{n}\right|}
\end{aligned}
$$

Since $\alpha\left|W \cap X_{n}\right|-1<\left\lfloor\alpha\left|W \cap X_{n}\right|\right\rfloor \leq \alpha\left|W \cap X_{n}\right|$ and $\left|X_{n}\right|$ is unbounded, we get that:

$$
\mu(V(\alpha))=\alpha \mu(W) .
$$




\section{Følner equivalence and Besicovitch submeasures}

\subsection{Følner equivalence}

Let $\left(X_{n}\right)$ and $\left(Y_{n}\right)$ be nondecreasing sequences of finite subsets of $G$. We say that they are synchronously Følner-equivalent if

$$
\lim _{n \rightarrow \infty} \frac{\left|X_{n} \Delta Y_{n}\right|}{\left|X_{n}\right|}=0
$$

Proposition 4.1. Consider nondecreasing sequences $\left(X_{n}\right)$ and $\left(Y_{n}\right)$. The following are equivalent.

1. $\left(X_{n}\right)$ and $\left(Y_{n}\right)$ are synchronously Følner-equivalent.

2. $\left|X_{n} \cap Y_{n}\right| \sim_{n \rightarrow \infty}\left|X_{n}\right| \sim_{n \rightarrow \infty}|Y|_{n}$.

3. $\left|X_{n}\right| \sim_{n \rightarrow \infty}\left|Y_{n}\right|$ and $\left|X_{n} \backslash Y_{n}\right|=o_{n \rightarrow \infty}\left(\left|X_{n}\right|\right)$.

Proof.

$1] 2$ This follows from the inequalities $|X| \geq|X \cap Y| \geq|X|-|X \Delta Y|$ and ||$X|-| Y|| \leq|X \Delta Y|$ which hold for every finite $X$ and $Y$.

2 2 3 Just note that $\left|X_{n} \backslash Y_{n}\right|=\left|X_{n}\right|-\left|X_{n} \cap Y_{n}\right|$.

3 $\Longrightarrow 1$ Note that:

$$
\begin{aligned}
\left|X_{n} \Delta Y_{n}\right| & =\left|X_{n} \backslash Y_{n}\right|+\left|Y_{n} \backslash X_{n}\right| \\
& =\left|X_{n} \backslash Y_{n}\right|+\left|Y_{n}\right|-\left|X_{n} \cap Y_{n}\right| \\
& =2\left|X_{n} \backslash Y_{n}\right|+\left|Y_{n}\right|-\left|X_{n}\right| \\
& =o_{n \rightarrow \infty}\left(\left|X_{n}\right|\right) .
\end{aligned}
$$

Corollary 4.2. Synchronous Følner equivalence is an equivalence relation.

Proof.

- From Proposition 4.1. note that if $\left(X_{n}\right)$ and $\left(Y_{n}\right)$ are synchronously Følner-equivalent, then $\frac{\left|X_{n} \Delta Y_{n}\right|}{\left|Y_{n}\right|} \sim_{n \rightarrow \infty} \frac{\left|X_{n} \Delta Y_{n}\right|}{\left|X_{n}\right|} \rightarrow_{n \rightarrow \infty} 0$. So the relation is symmetric.

- Transitivity follows from Proposition 4.1 and the inclusion $X \Delta Z \subseteq(X \Delta Y) \cup(Y \Delta Z)$, which holds for every $X, Y$ and $Z$.

- Reflexivity is trivial.

Since the definition involves a lim (and not a liminf), we immediately note the following.

Remark 4.3. $\left(X_{n}\right)$ and $\left(Y_{n}\right)$ are synchronously Følner-equivalent if and only if $\left(X_{k_{n}}\right)$ and $\left(Y_{k_{n}}\right)$ are synchronously Følner-equivalent, for every increasing sequence $\left(k_{n}\right)$.

We also denote $\left(X_{n}\right) \preceq\left(Y_{n}\right)$ if $\left(X_{n}\right)$ is synchronously Følner-equivalent to a subsequence $\left(Y_{m_{n}}\right)$. Equivalently,

$$
\lim _{n \rightarrow \infty} \min _{m \in \mathbb{N}} \frac{\left|X_{n} \Delta Y_{m}\right|}{\left|X_{n}\right|}=0 .
$$


To be convinced of the equivalence, note that the minimum is reached by some $m_{n}$ for each $n \in \mathbb{N}$, because $\left(Y_{m}\right)$ is nondecreasing and $X_{n}$ is finite. Thanks to symmetry of synchronous equivalence, we also have that $\left(X_{n}\right) \preceq\left(Y_{n}\right)$ if and only if $\lim _{n \rightarrow \infty} \min _{m \in \mathbb{N}} \frac{\left|X_{n} \Delta Y_{m}\right|}{\left|Y_{m}\right|}=0$. We say that they are Følner-equivalent, and write $\left(X_{n}\right) \sim\left(Y_{n}\right)$, if both $\left(X_{n}\right) \preceq\left(Y_{n}\right)$ and $\left(Y_{n}\right) \preceq\left(X_{n}\right)$. This is the case if they are synchronously Følner equivalent, but the converse is false. As counterexamples, one can consider twice the same sequence, but with repetitions on both sides that are longer and longer, and not synchronized. If one wants to obtain strictly increasing sequences, repetitions can be replaced by very slowly increasing sequences (point by point).

\section{Remark 4.4.}

1. $\preceq$ is a preorder relation.

2. Følner-equivalence is an equivalence relation.

Proof.

1. Reflexivity is obvious, and transitivity is not difficult.

2. Følner-equivalence is defined as the equivalence corresponding to a preorder, which is classical.

Proposition 4.5. Assume that $\left|X_{n}\right| \sim_{n \rightarrow \infty}\left|Y_{n}\right|$.

Then $\left(X_{n}\right)$ and $\left(Y_{n}\right)$ are synchronously Følner-equivalent if and only if $\left(X_{n}\right) \preceq\left(Y_{n}\right)$.

Proof. Assume $\left(X_{n}\right) \preceq\left(Y_{n}\right)$ (the converse implication is trivial). Let $n, m \in \mathbb{N}$. If $m \leq n$, then $\left|X_{n} \backslash Y_{n}\right| \leq\left|X_{n} \backslash Y_{m}\right|$ and $\left|Y_{n} \backslash X_{n}\right| \leq\left|Y_{n} \backslash Y_{m}\right|+\left|Y_{m} \backslash X_{n}\right|$ since $\left(Y_{n}\right)$ is nondecreasing. Summing up, $\left|X_{n} \Delta Y_{n}\right| \leq\left|X_{n} \Delta Y_{m}\right|+\left|Y_{n} \backslash Y_{m}\right|$. Symmetrically, if $n \leq m,\left|X_{n} \Delta Y_{n}\right| \leq$ $\left|X_{n} \Delta Y_{m}\right|+\left|Y_{m} \backslash Y_{n}\right|$. Overall for every $m \in \mathbb{N}$, we get $\left|X_{n} \Delta Y_{n}\right| \leq\left|X_{n} \Delta Y_{m}\right|+|| Y_{m}|-| Y_{n}||$. If we apply this with $\left(m_{n}\right)$ the subsequence from the definition of $\preceq$, which is such that $\left(X_{n}\right) \sim\left(Y_{m_{n}}\right)$, we have $\left|X_{n} \Delta Y_{m_{n}}\right|=o_{n \rightarrow \infty}\left(\left|X_{n}\right|\right)$, and by Proposition 4.1 (applied to $\left(X_{n}\right)$ and $\left(Y_{m_{n}}\right)$ ), $\left|Y_{m_{n}}\right| \sim_{n \rightarrow \infty}\left|X_{n}\right| \sim_{n \rightarrow \infty}\left|Y_{n}\right|$. Summing up, we deduce that $\left|X_{n} \Delta Y_{n}\right|=o_{n \rightarrow \infty}\left(\left|X_{n}\right|\right)$.

\subsection{Comparing Besicovitch submeasures}

A basic tool in our set constructions will be the following elementary remark.

Remark 4.6. If $\left(X_{n}\right)$ is nondecreasing and exhaustive, then for every finite set $W$ and every $\varepsilon>0$, there exists $n_{\left(X_{n}\right)}(W, \varepsilon)$ such that $\forall n \geq n_{\left(X_{n}\right)}(W, \varepsilon), \mathfrak{P}\left(W \mid X_{n}\right)<\varepsilon$ and $W \subset X_{n}$.

We deduce the following, which will be useful in our constructions.

Lemma 4.7. Let $\left(X_{n}\right)$ be a nondecreasing exhaustive sequence of an infinite group $G$. Let $W=\bigcup_{i \in \mathbb{N}} W_{i}$ where $\emptyset \neq W_{i} \Subset G$ for each $i \in \mathbb{N}$, such that, for every $n \in \mathbb{N}$, there are at most finitely many $i$ 's such that $W_{i} \cap X_{n} \neq \emptyset$ (this is the case, for example, if the $W_{i}$ 's are pairwise disjoint); in that case $j_{n}=\max _{W_{j} \cap X_{n} \neq \emptyset} j$ is well-defined for every $n$. Then:

1.

$$
\mu_{\left(X_{n}\right)}(W) \geq \limsup _{i \rightarrow \infty} \max _{m \in \mathbb{N}} \mathfrak{P}\left(W_{i} \mid X_{m}\right) .
$$

2. If there is a sequence $\left(\varepsilon_{n}\right)$ converging to 0 such that $\forall n \in \mathbb{N}, n_{\left(X_{n}\right)}\left(\bigcup_{i<j_{n}} W_{i}, \varepsilon_{n}\right) \leq n$, then:

$$
\mu_{\left(X_{n}\right)}(W)=\limsup _{i \rightarrow \infty} \max _{m \in \mathbb{N}} \mathfrak{P}\left(W_{i} \mid X_{m}\right)
$$


3. In general, there exists a nondecreasing integer sequence $\mathbf{l}$ such that, noting $W_{\mathbf{l}}=\bigcup_{i \in \mathbb{N}} W_{l_{i}}$ :

$$
\mu_{\left(X_{n}\right)}\left(W_{\mathbf{l}}\right)=\lim _{i \rightarrow \infty} \max _{m \in \mathbb{N}} \mathfrak{P}\left(W_{l_{i}} \mid X_{m}\right)
$$

Proof.

1. Let $\left(m_{i}\right)_{i \in \mathbb{N}}$ be a sequence of integers such that $\mathfrak{P}\left(W_{i} \mid X_{m_{i}}\right)=\max _{m \in \mathbb{N}} \mathfrak{P}\left(W_{i} \mid X_{m}\right)$. We know that this sequence goes to infinity (even though it may not be nondecreasing), because only finitely many $W_{i}$ 's intersect each $X_{m}$, but they all intersect at least one. Hence, $\mu_{\left(X_{n}\right)}(W)=\lim \sup _{n \rightarrow \infty} \mathfrak{P}\left(W \mid X_{n}\right) \geq \lim \sup _{i \rightarrow \infty} \mathfrak{P}\left(W \mid X_{m_{i}}\right)$. We get the desired inequality by noting that $W_{i} \subset W$.

2. Point 1 already gives one inequality. For the converse:

$$
\begin{aligned}
\mu_{\left(X_{n}\right)}(W) & =\limsup _{n \rightarrow \infty} \mathfrak{P}\left(\bigcup_{i<j_{n}} W_{i} \cup W_{j_{n}} \cup \bigcup_{i>j_{n}} W_{i} \mid X_{n}\right) \\
& \leq \limsup _{n \rightarrow \infty}\left(\mathfrak{P}\left(\bigcup_{i<j_{n}} W_{i} \mid X_{n}\right)+\mathfrak{P}\left(W_{j_{n}} \mid X_{n}\right)+\mathfrak{P}\left(\bigcup_{i>j_{n}} W_{i} \mid X_{n}\right)\right) \\
& \leq \limsup _{n \rightarrow \infty}\left(\varepsilon_{n}+\max _{m \in \mathbb{N}} \mathfrak{P}\left(W_{j_{n}} \mid X_{m}\right)+0\right) \\
& \leq \limsup _{n \rightarrow \infty}+\limsup _{n \rightarrow \infty} \max _{m \in \mathbb{N}} \mathfrak{P}\left(W_{j_{n}} \mid X_{m}\right) \\
& \leq 0+\limsup _{i \rightarrow \infty} \max _{m \in \mathbb{N}} \mathfrak{P}\left(W_{i} \mid X_{m}\right) .
\end{aligned}
$$

The last inequality comes from the fact that the sequence $\left(j_{n}\right)$ is nondecreasing (because $\left(X_{n}\right)$ is nondecreasing), and not upper-bounded (because the $W_{i}$ 's are nonempty), so it goes to infinity.

3. Let us define some sequence $\mathbf{l}$ by recurrence, from any seed $l_{0} \in \mathbb{N}$. Assume that $l_{n}$ is defined, and write $k_{n}=n_{\left(X_{n}\right)}\left(\bigcup_{j \leq n} W_{l_{j}}\right)$. Choose any $l_{n+1}$ such that for every $m \geq l_{n+1}$,

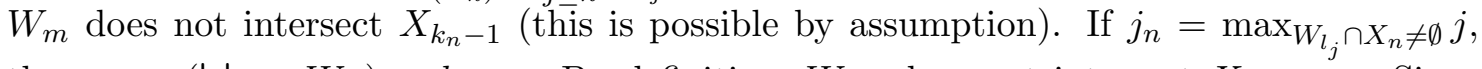
then $n_{\left(X_{n}\right)}\left(\bigcup_{j<j_{n}} W_{l_{j}}\right)=k_{j_{n}-1}$. By definition, $W_{l_{j_{n}}}$ does not intersect $X_{k_{j_{n}-1}-1}$. Since $W_{l_{n}}$ intersects $X_{n}$, we can deduce that $n>k_{j_{n}-1}-1$. This means that $\left(W_{l_{i}}\right)$ satisfies the hypothesis of Point 2 .

Replacing the limsup by a lim can be achieved by taking, again, a subsequence.

Lemma 4.8. Let $\varepsilon, \delta>0$, and $\left(X_{n}\right),\left(Y_{n}\right)$ be nondecreasing and exhaustive. The following are equivalent.

1. For every $W \subset G$, if $\mu_{\left(Y_{n}\right)}(W) \geq \varepsilon$, then $\mu_{\left(X_{n}\right)}(W) \geq \delta$.

2. $\lim \inf _{n \in \mathbb{N}} \max _{m \in \mathbb{N}} \frac{\varepsilon\left|Y_{n}\right|-\left|Y_{n} \backslash X_{m}\right|}{\left|X_{m}\right|} \geq \delta$.

If $m_{n}$ realizes the maximum for each $n \in \mathbb{N}$, and if $\varepsilon<1$, then these properties imply that

$$
\frac{\delta}{\varepsilon} \leq \liminf _{n \in \mathbb{N}} \frac{\left|Y_{n}\right|}{\left|X_{m_{n}}\right|} \leq \limsup _{n \in \mathbb{N}} \frac{\left|Y_{n}\right|}{\left|X_{m_{n}}\right|} \leq \frac{1-\delta}{1-\varepsilon} .
$$

In particular, the properties imply that $\delta \leq \varepsilon$. 
Proof.

- Let us start by proving the final inequalities. Suppose $\liminf \inf _{n \in \mathbb{N}} \frac{\varepsilon\left|Y_{n}\right|-\left|Y_{n} \backslash X_{m_{n}}\right|}{\left|X_{m_{n}}\right|} \geq \delta$.

Then on the one hand, it is clear that $\liminf _{n \in \mathbb{N}} \frac{\varepsilon\left|Y_{n}\right|}{\left|X_{m_{n}}\right|}$ is even bigger, which gives the first inequality. On the other hand, since $\left|Y_{n} \backslash X_{m_{n}}\right| \geq\left|Y_{n}\right|-\left|X_{m_{n}}\right|$, we can see that $\liminf \operatorname{in}_{n \in \mathbb{N}}(\varepsilon-1) \frac{Y_{n}}{X_{m_{n}}}+1 \geq \liminf _{n \in \mathbb{N}} \frac{\varepsilon\left|Y_{n}\right|-\left|Y_{n} \backslash X_{m_{n}}\right|}{\left|X_{m_{n}}\right|} \geq \delta$, which gives that $\lim \sup _{n \in \mathbb{N}} \frac{\left|Y_{n}\right|}{\left|X_{m_{n}}\right|} \leq$ $\frac{1-\delta}{1-\varepsilon}$, provided that $\varepsilon<1$.

$2 \nRightarrow 1$

$$
\begin{aligned}
\mu_{\left(X_{n}\right)}(W) & =\limsup _{m \rightarrow \infty} \mathfrak{P}\left(W \mid X_{m}\right) \\
& \geq \limsup _{n \rightarrow \infty} \mathfrak{P}\left(W \mid X_{m_{n}}\right) \\
& \geq \limsup _{n \rightarrow \infty} \frac{\left|W \cap Y_{n} \cap X_{m_{n}}\right|}{\left|X_{m_{n}}\right|} \\
& =\limsup _{n \rightarrow \infty} \frac{\left|W \cap Y_{n}\right|-\left|W \cap Y_{n} \backslash X_{m_{n}}\right|}{\left|X_{m_{n}}\right|} \\
& \geq \limsup _{n \rightarrow \infty} \frac{\left|W \cap Y_{n}\right|-\left|Y_{n} \backslash X_{m_{n}}\right|}{\left|X_{m_{n}}\right|} \\
& =\limsup _{n \rightarrow \infty}\left(\frac{\varepsilon\left|Y_{n}\right|-\left|Y_{n} \backslash X_{m_{n}}\right|}{\left|X_{m_{n}}\right|}+\frac{\left|W \cap Y_{n}\right|-\varepsilon\left|Y_{n}\right|}{\left|Y_{n}\right|} \frac{\left|Y_{n}\right|}{\left|X_{m_{n}}\right|}\right) \\
& \geq \liminf _{n \rightarrow \infty} \frac{\varepsilon\left|Y_{n}\right|-\left|Y_{n} \backslash X_{m_{n}}\right|}{\left|X_{m_{n}}\right|}+\left(\limsup _{n \rightarrow \infty} \frac{\left|W \cap Y_{n}\right|}{\left|Y_{n}\right|}-\varepsilon\right) \liminf _{n \in \mathbb{N}} \frac{\left|Y_{n}\right|}{\left|X_{m_{n}}\right|} \\
& \geq \delta+0 \frac{\delta}{\varepsilon} \text { by the two premises and the first inequalities. }
\end{aligned}
$$

$11 \Rightarrow 2$ Assume that $\liminf _{i \rightarrow \infty} \frac{\varepsilon\left|Y_{i}\right|-\left|Y_{i} \backslash X_{k_{i}}\right|}{\left|X_{k_{i}}\right|}<\delta$. Let us build a set $W$ that contradicts Point 1 . For each $n \in \mathbb{N}$, there exists $k_{n}=\min \left\{k|| Y_{n} \backslash X_{k}|\leq \varepsilon| Y_{n} \mid\right\}$, because for large $k, Y_{n} \backslash X_{k}=$ $\emptyset$ (because $\left(X_{k}\right)$ is exhaustive and $Y_{n}$ is finite). By noting that $\left(Y_{n} \cap X_{k_{n}}\right) \backslash X_{k_{n}-1}=\left(Y_{n} \backslash\right.$ $\left.X_{k_{n}-1}\right) \backslash\left(Y_{n} \backslash X_{k_{n}}\right)$ (by convention $X_{-1}$ is empty), we can write that $\left|\left(Y_{n} \cap X_{k_{n}}\right) \backslash X_{k_{n}-1}\right|=$ $\left|Y_{n} \backslash X_{k_{n}-1}\right|-\left|Y_{n} \backslash X_{k_{n}}\right|$, which is bigger than $\varepsilon\left|Y_{n}\right|-\left|Y_{n} \backslash X_{k_{n}}\right|$, by minimality of $k_{n}$. Hence $\left(Y_{n} \cap X_{k_{n}}\right) \backslash X_{k_{n}-1}$ admits a subset $Z_{n}$ of cardinality $\left|Z_{n}\right|=\left\lfloor\varepsilon\left|Y_{n}\right|\right\rfloor-\left|Y_{n} \backslash X_{k_{n}}\right|$. Define $W_{n}=\left(Y_{n} \backslash X_{k_{n}}\right) \bigsqcup Z_{n}$. Note that $W_{n} \subset Y_{n}$, and that $\varepsilon-\frac{1}{\left|Y_{n}\right|}<\mathfrak{P}\left(W_{n} \mid Y_{n}\right) \leq \varepsilon$. The $W_{i}$ satisfy the hypotheses of Lemma 4.7, so that Point 3 gives an integer sequence $\mathbf{l}$, with $\mu_{\left(X_{n}\right)}\left(W_{\mathbf{l}}\right)=\lim _{i \rightarrow \infty} \max _{m \in \mathbb{N}} \mathfrak{P}\left(W_{l_{i}} \mid X_{m}\right)$. By construction, we have:

$$
\begin{aligned}
\mathfrak{P}\left(W_{i} \mid X_{m}\right) & =\mathfrak{P}\left(Y_{i} \backslash X_{k_{i}} \mid X_{m}\right)+\mathfrak{P}\left(Z_{i} \mid X_{m}\right) \\
& =\frac{\left|Y_{i} \cap X_{m} \backslash X_{k_{i}}\right|+\left|Z_{i} \cap X_{m}\right|}{\left|X_{m}\right|} .
\end{aligned}
$$

If $m<k_{i}$, then $X_{m} \subseteq X_{k_{i}}$, and $Z_{i} \cap X_{m} \subseteq Z_{i} \cap X_{k_{i}-1}=\emptyset$, so that this quantity is 0 . On the contrary, if $m \geq k_{i}$, then $Z_{i} \subseteq X_{k_{i}} \subseteq X_{m}$, and $Y_{i} \cap X_{m} \backslash X_{k_{i}}=\left(Y_{i} \backslash X_{k_{i}}\right) \backslash\left(Y_{i} \backslash X_{m}\right)$, 
so that:

$$
\begin{aligned}
\mathfrak{P}\left(W_{i} \mid X_{m}\right) & =\frac{\left|Y_{i} \cap X_{m} \backslash X_{k_{i}}\right|+\left|Z_{i} \cap X_{m}\right|}{\left|X_{m}\right|} \\
& =\frac{\left|Y_{i} \cap X_{m} \backslash X_{k_{i}}\right|+\left|Z_{i}\right|}{\left|X_{m}\right|} \\
& =\frac{\left|Y_{i} \backslash X_{k_{i}}\right|-\left|Y_{i} \backslash X_{m}\right|+\left\lfloor\varepsilon\left|Y_{i}\right|\right\rfloor-\left|Y_{i} \backslash X_{k_{i}}\right|}{\left|X_{m}\right|} \\
& \leq \max _{m \in \mathbb{N}} \frac{\left|\left\lfloor\varepsilon\left|Y_{i}\right|\right\rfloor\right|-\left|Y_{i} \backslash X_{m}\right|}{\left|X_{m}\right|} \\
& <\delta \text { by hypothesis. }
\end{aligned}
$$

Taking the limit, we get that $\mu_{\left(X_{n}\right)}\left(W_{\mathbf{l}}\right)<\delta$.

On the other hand, applying now Point 1 of Lemma 4.7 to sequence $\left(Y_{n}\right)$ :

$$
\mu_{\left(Y_{n}\right)}\left(W_{\mathbf{l}}\right) \geq \lim _{i \in \mathbb{N}} \max _{m \in \mathbb{N}} \mathfrak{P}\left(W_{l_{i}} \mid Y_{m}\right) \geq \mathfrak{P}\left(W_{l_{i}} \mid Y_{l_{i}}\right)=\varepsilon
$$

The previous lemma now allows to characterize the main properties of interest for comparing two Besicovitch submeasures.

Proposition 4.9. Let $\left(X_{n}\right)$ and $\left(Y_{n}\right)$ be nondecreasing and exhaustive.

1. $\mu_{\left(Y_{n}\right)}$ is $\lambda$-Lipschitz with respect to $\mu_{\left(X_{n}\right)}$, where $\lambda>0$, if and only if

$$
\forall \varepsilon>0, \liminf _{n \rightarrow \infty} \max _{m \in \mathbb{N}} \frac{\left|Y_{n}\right|-\frac{1}{\varepsilon}\left|Y_{n} \backslash X_{m}\right|}{\left|X_{m}\right|} \geq \frac{1}{\lambda}
$$

2. $\mu_{\left(Y_{n}\right)}$ is absolutely continuous with respect to $\mu_{\left(X_{n}\right)}$ if and only if it is Lipschitz.

3. $\mu_{\left(Y_{n}\right)} \leq \mu_{\left(X_{n}\right)}$ if and only if $\left(Y_{n}\right) \preceq\left(X_{n}\right)$.

4. $\mu_{\left(Y_{n}\right)}=\mu_{\left(X_{n}\right)}$ if and only if $\left(Y_{n}\right) \sim\left(X_{n}\right)$.

One can even see from the proof that $\left(Y_{n}\right) \preceq\left(X_{n}\right)$ if and only if there exists $\left.\varepsilon \in\right] 0,1[$ such that $\forall W \subset G, \mu_{\left(X_{n}\right)}(W)<\varepsilon \Longrightarrow \mu_{\left(Y_{n}\right)}(W)<\varepsilon$.

Proof.

1. Just note that the $\lambda$-Lipschitz property of $\mu_{\left(Y_{n}\right)}$ is equivalent to the properties in Lemma 4.8, for every $\delta$ and $\varepsilon=\lambda \delta$, and hence to:

$$
\liminf _{n \in \mathbb{N}} \max _{m \in \mathbb{N}} \frac{\left|Y_{n}\right|-\frac{1}{\varepsilon}\left|Y_{n} \backslash X_{m}\right|}{\left|X_{m}\right|} \geq \frac{1}{\lambda} .
$$

2. From Lemma 4.8, $\mu_{\left(Y_{n}\right)}$ is absolutely continuous with respect to $\mu_{\left(X_{n}\right)}$ if and only if

$$
\forall \varepsilon>0, \liminf _{n \rightarrow \infty} \max _{m \in \mathbb{N}} \frac{\left|Y_{n}\right|-\frac{1}{\varepsilon}\left|Y_{n} \backslash X_{m}\right|}{\left|X_{m}\right|}>0 .
$$

From Point 1, this is equivalent to the existence of some $\lambda$ such that $\mu_{\left(Y_{n}\right)}$ is $\lambda$-Lipschitz with respect to $\mu_{\left(X_{n}\right)}$. 
3. Consider a sequence $\left(m_{n}\right)$ which witnesses that $\left(Y_{n}\right) \preceq\left(X_{n}\right): \lim _{n \rightarrow \infty} \frac{\left|Y_{n} \Delta X_{m_{n}}\right|}{\left|Y_{n}\right|}=0$. Then

$$
\begin{gathered}
\lim _{n \in \mathbb{N}} \frac{\left|Y_{n}\right|-\frac{1}{\varepsilon}\left|Y_{n} \backslash X_{m_{n}}\right|}{\left|X_{m_{n}}\right|}=\lim _{n \in \mathbb{N}} \frac{\left|Y_{n}\right|}{\left|X_{m_{n}}\right|}\left(1-\frac{1}{\varepsilon} \lim _{n \in \mathbb{N}} \frac{\left|Y_{n} \backslash X_{m_{n}}\right|}{\left|Y_{n}\right|}\right) \\
=1 .
\end{gathered}
$$

We can conclude by Point 1 .

Conversely, suppose that

$$
\liminf _{n \in \mathbb{N}} \frac{\left|Y_{n}\right|-\frac{1}{\varepsilon}\left|Y_{n} \backslash X_{m_{n}}\right|}{\left|X_{m_{n}}\right|} \geq 1
$$

By the last inequalities in Lemma 4.8 , we know that $\lim _{n \in \mathbb{N}} \frac{\left|Y_{n}\right|}{\left|X_{m_{n}}\right|}=1$. Moreover,

$$
\lim _{n \rightarrow \infty} \frac{\left|Y_{n} \backslash X_{m_{n}}\right|}{\left|X_{m_{n}}\right|} \leq \lim _{n \rightarrow \infty} \frac{\varepsilon\left|Y_{n}\right|}{\left|X_{m_{n}}\right|}-\varepsilon \liminf _{n \in \mathbb{N}} \frac{\left|Y_{n}\right|-\frac{1}{\varepsilon}\left|Y_{n} \backslash X_{m_{n}}\right|}{\left|X_{m_{n}}\right|}=\varepsilon-\varepsilon=0 .
$$

By Point 3 of Proposition 4.1, we obtain that $\left(Y_{n}\right) \preceq\left(X_{n}\right)$.

4. This is direct from the definitions and the Point 3 .

The following is direct from Proposition 4.9 and Remark 2.3 .

Corollary 4.10. Let $\left(X_{n}\right)$ and $\left(Y_{n}\right)$ be nondecreasing and exhaustive. Then $\left(Y_{n}\right) \preceq\left(X_{n}\right)$ (resp. $\left.\left(Y_{n}\right) \sim\left(X_{n}\right)\right)$ if and only if the identity map from space $A^{G}$ endowed with $d_{\left(X_{n}\right)}$ onto space $A^{G}$ endowed with $d_{\left(Y_{n}\right)}$ is 1-Lipschitz (resp. an isometry).

Here are particular classes of sequences, where the proposition can be applied.

Corollary 4.11. Let $\left(X_{n}\right)$ and $\left(Y_{n}\right)$ be nondecreasing and exhaustive.

1. If there exist a real number $\lambda>0$ and a sequence $\left(m_{n}\right)$ such that $\lim _{n \rightarrow \infty} \inf _{n \rightarrow \infty} \mathfrak{P}\left(X_{n} \mid Y_{m_{n}}\right) \geq$ $\frac{1}{\lambda}$ and $X_{n} \subset Y_{m_{n}}$, then $\mu_{\left(X_{n}\right)}$ is $\lambda$-Lipschitz with respect to $\mu_{\left(Y_{n}\right)}$.

2. If for cofinitely many $n \in \mathbb{N}, Y_{n} \subset X_{n+1}$ and $\liminf _{n \rightarrow \infty} \mathfrak{P}\left(X_{n} \mid X_{n+1}\right) \geq \lambda$, then $\mu_{\left(X_{n}\right)}$ is $\lambda$-Lipschitz with respect to $\mu_{\left(Y_{n}\right)}$.

3. On the other hand, if $\left|X_{n}\right| \sim_{n \rightarrow \infty}\left|Y_{n}\right|$ but $\left(X_{n}\right)$ and $\left(Y_{n}\right)$ are not (synchronously) Følnerequivalent, and $n_{\left(Y_{m}\right)}\left(X_{n}, \varepsilon_{n}\right)=n+1$ for some real sequence $\left(\varepsilon_{n}\right)$ converging to 0 , then $\mu_{\left(X_{n}\right)}$ is not absolutely continuous with respect to $\mu_{\left(Y_{n}\right)}$.

Proof.

1. For every $\varepsilon>0$,

$$
\liminf _{n \rightarrow \infty} \max _{m \in \mathbb{N}} \frac{\left|X_{n}\right|-\frac{1}{\varepsilon}\left|X_{n} \backslash Y_{m}\right|}{\left|Y_{m}\right|} \geq \liminf _{n \rightarrow \infty} \frac{\left|X_{n}\right|-\frac{1}{\varepsilon}\left|X_{n} \backslash Y_{m_{n}}\right|}{\left|Y_{m_{n}}\right|}=\liminf _{n \rightarrow \infty} \frac{\left|X_{n}\right|}{\left|Y_{m_{n}}\right|} \geq \frac{1}{\lambda} .
$$

2. Apply Point 1 with $m_{n}=\min \left\{m \in \mathbb{N} \mid X_{n} \subset Y_{m}\right\}$; the hypothesis is that $m_{n}$ is ultimately $n+1$. 
3. Suppose $\left|X_{n}\right| \sim_{n \rightarrow \infty}\left|Y_{n}\right|$ and $\left(X_{n}\right)$ and $\left(Y_{n}\right)$ are not synchronously Følner-equivalent. By Proposition 4.5. $\left(X_{n}\right) \npreceq\left(Y_{n}\right)$, that is, $\varepsilon=\limsup _{n \rightarrow \infty} \frac{\left|X_{n} \backslash Y_{n}\right|}{\left|Y_{n}\right|}>0$. We can write $\liminf _{n \rightarrow \infty} \frac{\left|X_{n}\right|-\frac{1}{\varepsilon}\left|X_{n} \backslash Y_{n}\right|}{\left|Y_{n}\right|}=0$.

By the second assumption, for every $m>n, X_{n} \backslash Y_{m}=\emptyset$ and $\frac{\left|X_{n}\right|}{\left|Y_{m}\right|} \leq \varepsilon_{n}$. We get:

$$
\max _{m \in \mathbb{N}} \frac{\left|X_{n}\right|-\frac{1}{\varepsilon}\left|X_{n} \backslash Y_{m}\right|}{\left|Y_{m}\right|} \leq \max \left(\frac{\left|X_{n}\right|-\frac{1}{\varepsilon}\left|X_{n} \backslash Y_{n}\right|}{\left|Y_{n}\right|}, \varepsilon_{n}\right) .
$$

Putting things together, $\lim \inf _{n \rightarrow \infty} \max _{m \in \mathbb{N}} \frac{\left|X_{n}\right|-\frac{1}{\varepsilon}\left|X_{n} \backslash Y_{m}\right|}{\left|Y_{m}\right|}$ is 0 . We conclude by Point 2 of Proposition 4.9 .

Corollary 4.12. Let $\left(X_{n}\right)$ and $\left(Y_{n}\right)$ be nondecreasing and exhaustive. Assume that $\left|X_{n}\right| \sim_{n \rightarrow \infty}$ $\left|Y_{n}\right|$. Then the following are equivalent.

1. $\left(X_{n}\right)$ and $\left(Y_{n}\right)$ are synchronously Følner-equivalent.

2. $\mu_{\left(Y_{l_{n}}\right)}=\mu_{\left(X_{l_{n}}\right)}$, for every increasing sequence $\left(l_{n}\right) \in \mathbb{N}^{\mathbb{N}}$.

3. $\mu_{\left(Y_{l_{n}}\right)}$ is absolutely continuous with respect to $\mu_{\left(X_{l_{n}}\right)}$, for every increasing sequence $\left(l_{n}\right)$.

Proof.

$11 \Longrightarrow 2$ By Remark 4.3 , synchronous Følner equivalence is transmitted to all subsequences (provided that one takes the same subsequence for $\left(X_{n}\right)$ and for $\left(Y_{n}\right)$ ). We conclude thanks to Proposition 4.9 .

$2 \Longrightarrow 3$ This is obvious.

1 $\Longrightarrow$ If $\left(X_{n}\right)$ and $\left(Y_{n}\right)$ are not synchronously Følner-equivalent, then there exists an infinite set $I \subset \mathbb{N}$ and a real number $\alpha>0$ such that $\forall n \in I, \frac{\left|X_{n} \Delta Y_{n}\right|}{\left|X_{n}\right|} \geq \alpha$. This implies that for every increasing sequence $\left(l_{n}\right) \in I^{\mathbb{N}},\left(X_{l_{n}}\right)$ and $\left(Y_{l_{n}}\right)$ are not synchronously Følner-equivalent. We can take an increasing sequence $\left(l_{n}\right) \in I^{\mathbb{N}}$ such that $n_{\left(Y_{m}\right)}\left(X_{l_{n}}, \varepsilon_{l_{n}}\right)=l_{n+1}$, for some real sequence $\left(\varepsilon_{n}\right)$ converging to 0 . Then $\left(X_{l_{n}}\right)$ and $\left(Y_{l_{n}}\right)$ satisfy the assumptions for Point 3 of Corollary 4.11 .

\subsection{Shift}

If $G$ is a group and $\left(X_{n}\right) \sim\left(g X_{n}\right)$, then we say that $\left(X_{n}\right)$ is (left) $g$-Følner. Since $\left|X_{n}\right|=\left|g X_{n}\right|$, Proposition 4.5 says that it is enough to require $\left(X_{n}\right) \preceq\left(g X_{n}\right)$, and that in this case, $\left(X_{n}\right)$ and $\left(g X_{n}\right)$ are synchronously Følner-equivalent.

A (left) Følner sequence for a countable group $G$ is a $g$-Følner sequence for every $g \in G$. A countable group is amenable if and only if it admits a Følner sequence: cf. CSC10, Chapter 4], in particular for many alternative definitions.

A group $G$ is finitely generated (briefly, f.g.) if $E \Subset G$ exists such that for every $g \in G$ there exists $e_{1}, \ldots, e_{n} \in E \cup E^{-1}$ such that $e_{1} \cdots e_{n}=g$. Remarkably (cf. [Pet, Lemma 5.3]) if a f.g. group is amenable, then it has a nondecreasing exhaustive Følner sequence. In addition, if the size of the balls grows polynomially with the radius, then they form a Følner sequence, so Point 3 of Corollary 4.13 generalizes [HM17, Cor 4.1.4].

The following is a rephrasing of Corollary 4.10 .

Corollary 4.13. Let $G$ be a countable group and let $\left(X_{n}\right)$ be a nondecreasing exhaustive sequence. 
1. ( $\left.X_{n}\right)$ is $g$-Følner if and only if $\mu_{\left(X_{n}\right)}=\mu_{\left(g^{-1} X_{n}\right)}$ if and only if the shift by $g$ is an isometry.

2. $\left(X_{n}\right)$ is Følner if and only if every shift is an isometry.

3. If $G$ is finitely generated, then $G$ is amenable if and only if there exists a nondecreasing sequence $\left(X_{n}\right)$ of finite subsets of $G$ such that every shift is an isometry.

Note that one implication of Point 3 was already stated in Cap09, Theorem 3.5], but the proof contains a confusion between left and right Følner. The full equivalence generalizes [HM17, Cor 4.1.4] (since balls are Følner in polynomial-growth groups).

Corollary 4.14. Let $G$ be a finitely generated group.

1. If $\left(X_{n}\right)$ is the sequence of balls with respect to some generating set of cardinality $\alpha$, then every shift is $\alpha$-Lipschitz.

2. If $g \in G$, a nondecreasing exhaustive sequence is $g$-Følner if and only if all of its subsequences yield a Besicovitch pseudodistance for which the shift by $g$ is continuous.

3. $G$ is amenable if and only if it admits a nondecreasing exhaustive sequence of finite subsets of which all subsequences yield a Besicovitch distance for which every shift is continuous.

The first point generalizes [HM17, Prop 4.1.3]. Note that it still applies in nonamenable groups, but the shifts are no longer isometries, and there is a subsequence of balls with respect to which the Besicovitch pseudodistance makes them non-continuous.

We remark (cf. dlH00, VII.34]) that the sequence of balls is Følner if and only if the group has polynomial growth, and has a Følner subsequence if and only if it has subexponential growth.

Proof.

1. If $E$ is the generating set and $E_{n}$ the corresponding radius-n ball, then $E_{0}=\{e\}$ where $e$ is the identity of $G$ and $E_{n+1}=\left(E \cup E^{-1}\right) \cdot E_{n}$, so $\left|E_{n}\right| \leq(2|E|+1)^{n}$. We can apply Point 2 of Corollary 4.11 .

2. This comes from Corollary 4.12

3. This comes from Point 2 and the characterization of amenability through Følner sequences.

There are nondecreasing non-Følner sequences for which the shift is Lipschitz (but not an isometry) in $\mathbb{Z}^{d}$. Here's an example: $X_{n}=(\llbracket-n, n \rrbracket \cup 2 \rrbracket-n, n \llbracket)^{d}$. Indeed, for every $n$, $1+X_{n} \subset X_{2 n}$ and $\frac{\left|X_{2 n}\right|}{\left|X_{n}\right|}=\frac{(8 n-1)^{d}}{(4 n-1)^{d}}$, which converges to $2^{d}$ when $n$ goes to infinity. We conclude by Point 1 of Corollary 4.11, with $m_{n}=2 n$ and $\alpha=2^{d}$. But the shift is not an isometry because the sequence is not Følner: $\mu\left((2 \mathbb{Z})^{d}\right)=2^{d} / 3^{d}>\mu\left((2 \mathbb{Z}+1)^{d}\right)=1 / 3^{d}$.

\subsection{Propagations and right Følner sequences}

Let $G$ be a group and let $g \in G$. A sequence $\left(X_{n}\right)$ of finite subsets of $G$ is right $g$-Følner if $\left(X_{n}\right) \sim\left(X_{n} g\right)$; equivalently, if $\left(X_{n}^{-1}\right)$ is left $g^{-1}$-Følner. A right Følner sequence is then a sequence which is right $g$-Følner for every $g \in G$.

Let now $A$ be an alphabet. The propagation in direction $g \in G$ is the function $\pi^{g}: A^{G} \rightarrow A^{G}$ defined by $\pi^{g}(x)(i)=x\left(i g^{-1}\right)$ for every $x \in A^{G}$ and $i \in G$. With this definition, the value of $\pi^{g}(x)$ at point $i g$ equals the value of $x$ at point $i$ : that is, the information moves in direction g. Points 1, 2 and 3 of Corollary 4.13 can then be dualized to right Følner sequences and propagations: 
Corollary 4.15. Let $G$ be a group.

1. A nondecreasing exhaustive sequence $\left(X_{n}\right)$ is right $g$-Følner if and only if $\mu_{\left(X_{n}\right)}=\mu_{\left(X_{n} g^{-1}\right)}$ if and only if the propagation in direction $g$ is an isometry.

2. A nondecreasing exhaustive sequence is right Følner if and only if every propagation is an isometry.

3. A finitely generated group is amenable if and only if there exists a nondecreasing exhaustive sequence $\left(X_{n}\right)$ of finite subsets of $G$ such that every propagation is an isometry.

Proof. The first equivalence of Point 1 is immediate. For the other one, given $x \in A^{G}$, let $\bar{x}(i)=$ $x\left(i^{-1}\right)$ for every $x \in A^{G}$ and $i \in G$ : then for every $x, y \in A^{G}$ and $g \in G$ it is $\overline{\bar{x}}=x, \Delta(\bar{x}, \bar{y})=$ $(\Delta(x, y))^{-1}$ and $\overline{\pi^{g}(x)}=\sigma^{g}(\bar{x})$, thus also $d_{\left(X_{n}\right)}(x, y)=d_{\left(X_{n}^{-1}\right)}(\bar{x}, \bar{y})$ and $d_{\left(X_{n}\right)}\left(\pi^{g}(x), \pi^{g}(y)\right)=$ $d_{\left(X_{n}^{-1}\right)}\left(\sigma^{g}(\bar{x}), \sigma^{g}(\bar{y})\right)$, so that the propagation in direction $g$ is an isometry for $d_{\left(X_{n}\right)}$ if and only if the shift by $g$ is an isometry for $d_{X_{n}^{-1}}$. Points 2 and 3 follow easily.

\subsection{Block maps}

A block map on a group $G$ with source alphabet $A$, target alphabet $B$, neighborhood $N=$ $\left\{j_{1}, \ldots, j_{k}\right\}$ and local rule $\phi: A^{k} \rightarrow B$ is a function $F: A^{G} \rightarrow B^{G}$ defined as the synchronous application of $\phi$ at the " $N$-shaped neighborhood" of each point of the group: that is, for every $x \in A^{G}$ and $i \in G, F(x)(i)=\phi\left(x\left(i j_{1}\right), \ldots, x\left(i j_{n}\right)\right)$. By the Curtis-Lyndon-Hedlund theorem Hed69] (see also [CSC10, Chapter 1]), block maps are all and only those functions from $A^{G}$ to $B^{G}$ which are continuous in the prodiscrete topology and commute with all the shifts. Every propagation is a block map, but the shift by $g \in G$ is a block map if and only if $g$ is central in $G$, that is, $g h=h g$ for every $h \in G$; in this case, $\sigma^{g}=\pi^{g^{-1}}$. Note that the local rule $\phi$ itself can be identified with a block map $\Phi$ with source alphabet $A^{k}$, target alphabet $B$, and neighborhood $N=\{e\}$, where $e$ is the identity element of $G$. Such a function is surely 1-Lipschitz, but not necessarily an isometry: for example, $\phi$ could be constant.

Block maps can be defined equivalently as follows. For $f_{1}, \ldots, f_{k}: A^{G} \rightarrow A^{G}$ define the product $f=f_{1} \times \ldots \times f_{k}: A^{G} \rightarrow\left(A^{k}\right)^{G}$ by $f(x)(i)=\left(f_{1}(x)(i), \ldots, f_{k}(x)(i)\right)$ for every $x \in A^{G}$ and $i \in G$. Then a block map $F$ with source alphabet $A$, target alphabet $B$, neighborhood $N=\left\{j_{1}, \ldots, j_{k}\right\}$ and local rule $\phi$ has the form $F=\Phi \circ\left(\pi^{j_{1}^{-1}} \times \ldots \times \pi^{j_{k}^{-1}}\right)$, where $\Phi$ is as in the previous paragraph.

Lemma 4.16. Suppose $f_{1}, \ldots, f_{k}: A^{G} \rightarrow A^{G}$ are such that $f_{q}$ is $\alpha_{q}$-Lipschitz with respect to $d_{\left(X_{n}\right)}$. Then $f=f_{1} \times \ldots \times f_{k}: A^{G} \rightarrow\left(A^{k}\right)^{G}$ is $\left(\sum_{q=1}^{k} \alpha_{q}\right)$-Lipschitz with respect to $d_{\left(X_{n}\right)}$. In particular, if $\alpha_{q}=\alpha$ for every $q \in[1: k]$, then $f$ is $k \alpha$-Lipschitz, and if each $f_{q}$ is an isometry, then $f$ is $k$-Lipschitz.

Proof. For every $x, y \in A^{G}$ and $i \in G$, we have $f(x)(i) \neq f(y)(i)$ if and only if $f_{q}(x)(i) \neq f_{q}(y)(i)$ for at least one $q \in[1: k]$, that is, $\Delta(f(x), f(y))=\bigcup_{q=1}^{k} \Delta\left(f_{q}(x), f_{q}(y)\right)$. Consequently, for every $n \geq 1$, we have $\left|\Delta(f(x), f(y)) \cap X_{n}\right| \leq \sum_{q=1}^{k}\left|\Delta\left(f_{i}(x), f_{i}(y)\right) \cap X_{n}\right|$, and the thesis follows easily.

The composition of an $\alpha$-Lipschitz function with a $\beta$-Lipschitz function is an $\alpha \beta$-Lipschitz function. As every propagation is a block map, Lemma 4.16 and Point 2 of Corollary 4.15 allow us to dualize Points 2 and 3 of Corollary 4.14 and recover (cf. [Cap09, Theorem 3.7] and [Cap11, Theorem 18]) the following characterization.

\section{Corollary 4.17.}


1. A nondecreasing exhaustive sequence of finite subsets of a group $G$ is right Følner if and only if all of its subsequences yield a Besicovitch pseudodistance for which every block map is continuous.

2. A finitely generated group is amenable if and only if it admits a nondecreasing exhaustive sequence $\left(X_{n}\right)$ of finite subsets such that, for every $k \geq 1$ and every increasing $\left(l_{n}\right) \in \mathbb{N}^{\mathbb{N}}$, every block map with neighborhood size $k$ is $k$-Lipschitz with respect to $d_{\left(X_{l_{n}}\right)}$.

\section{Conclusions}

We have presented a way to compare Besicovitch submeasures (in terms of absolute continuity, Lipschitz continuity, equality) thanks to the sequences of finite sets which describe them. In a shift space (with respect to a countable group) endowed with the Besicovitch topology, we have derived conditions on the defining sequence for the shift maps to be continuous, Lipschitz or isometric. As part of this, we gave another characterization of amenable groups.

Possible future work could involve extension to configuration spaces on possibly uncountable groups. This would require the use of the more general notions of directed set and of net, and although the definition of Besicovitch pseudodistance and submeasure would be immediate to extend, the techniques used to prove the main lemmas could need a major revision.

\section{References}

[BFK99] François Blanchard, Enrico Formenti, and Petr Kưrka. Cellular automata in the Cantor, Besicovitch and Weyl spaces. Complex Systems, 11:107-123, 1999.

[Cap09] Silvio Capobianco. Surjunctivity for cellular automata in Besicovitch spaces. Journal of Cellular Automata, 4:89-98, 2009.

[Cap11] Silvio Capobianco. Generalized Besicovitch and Weyl spaces: Topology, patterns, and sliding block codes. Theoretical Computer Science, 412:3822-3837, 2011.

[CGK13] Silvio Capobianco, Pierre Guillon, and Jarkko Kari. Surjective cellular automata far from the Garden of Eden. Discrete Mathematics and Theoretical Computer Science, 15:41-60, 2013.

[CSC10] Tullio G. Ceccherini-Silberstein and Michel Coornaert. Cellular Automata and Groups. Springer Verlag, 2010.

[dlH00] Pierre de la Harpe. Topics in Geometric Group Theory. The University of Chicago Press, 2000.

[Hed69] Gustav A. Hedlund. Endomorphisms and automorphisms of the shift dynamical system. Mathematical Systems Theory, 3:320-375, 1969.

[HM17] Karl-Peter Hadeler and Johannes Müller. Cellular Automata : Analysis and Applications. Springer, 2017. 00000.

[Pet] Gábor Pete. Probability and geometry on groups. Lecture notes. May 19, 2019. http://math.bme.hu/ gabor/PGG.pdf.

[Sab06] Mathieu Sablik. Étude de l'action conjointe d'un automate cellulaire et du décalage: une approche topologique et ergodique. PhD thesis, Université de Provence, July 2006. 